\title{
Simultaneous Localization and Mapping in Multipath Environments: Mapping and Reusing of Virtual Transmitters
}

\author{
Christian Gentner, Boxiao Ma, Robert Pöhlmann, Markus Ulmschneider, Thomas Jost, Armin Dammann \\ German Aerospace Center (DLR) \\ Institute of Communications and Navigation \\ Oberpfaffenhofen, 82234 Wessling, Germany \\ Email: \{Christian.Gentner, Boxiao.Ma, Robert.Poehlmann, Markus.Ulmschneider, Thomas.Jost, Armin.Dammann\}@ dlr.de
}

\begin{abstract}
BIOGRAPHY
Christian Gentner studied electrical engineering at the University of Applied Science in Ravensburg, with the main topic communication technology and received his Dipl.-Ing. (BA) degree in 2006. During this study he received practical experiences at Rohde \& Schwarz in Munich. He continued his study at the University of Ulm until 2009, where he received his M.Sc. degree. He is currently working towards the Ph.D. degree at the Institute of Communications and Navigation of the German Aerospace Center (DLR). His current research focuses on multipath assisted positioning.
\end{abstract}

Boxiao Ma studied electrical engineering with emphasis in telecommunication at the Southeast University in Nanjing and received his Bachelor degree in 2013. Directly after that he was pursuing his Master degree at the Technical University of Munich and he got the degree in 2016. He also collected practical experience from Internship in ZTE Corporation and German Aerospace Center In 2016 he joined the Signal and Information Processing Laboratory at ETH Zurich. His research interests lie in extracting information from signals of almost any kind.

Robert Pöhlmann is studying electrical engineering with focus on communications engineering at the Technical University of Munich (TUM). He received his B.Sc. degree in 2014 and his Master's degree in 2016. In 2013 he joined the Institute of Communications and Navigation of the German Aerospace Center (DLR) as a student trainee. The main topic of his current work is multipath tracking and multipath assisted positioning.

Markus Ulmschneider studied communications and computer engineering at the University of Ulm, Germany, from where he received his Bachelor's degree in 2011 and his Master's degree in 2014. In 2014, he joined the Institute of Communications and Navigation of the German Aerospace Center (DLR), Germany, where he is part of the scientific staff of the Mobile Radio Transmission group. His main research interests include multipath assisted positioning as well as multi-sensor localization and tracking techniques.

Thomas Jost received a Diploma degree (FH) 2001 in Electrical Engineering from University of Applied Science Wiesbaden, Germany and Diploma degree 2003 in Electrical Engineering and Information Technology from Technical University of Darmstadt, Germany. From 2003 to 2006 he held a research assistant position at Signal Processing Group at TU Darmstadt. Since 2006 he is a member of the scientific staff of the Institute of Communications and Navigation at the German Aerospace Center (DLR).

Armin Dammann studied electrical engineering at the University of Ulm, Germany, with main topic informationand microwave-technology. He received the Dipl.-Ing. and Dr.-Ing. (PhD) degree in 1997 and 2005 respectively, both from the University of Ulm. In 1997 Armin Dammann joined the Institute of Communications and Navigation of the German Aerospace Center (DLR). Since 2005 he is head of the Mobile Radio Transmission research Group. His research focus currently includes synchronization/positioning for terrestrial OFDM systems.

Abstract-Channel-SLAM is an algorithm which uses multipath propagation for positioning and treats multipath components (MPCs) as line-of-sight (LoS) signals originated from virtual transmitters (VTs). To use the information of the MPCs, Channel-SLAM estimates the receiver position and the position of the VTs simultaneously using simultaneous localization and mapping (SLAM) and does not require any prior information such as room-layout or a database for fingerprinting. This paper investigates mapping, where we derive a probabilistic map representation based on the receiver positions. Thus, if the receiver knows its current location, the information in the probabilistic map helps to estimate the trajectory of further receiver movement. Similar to SLAM approaches, we estimate, map and reuse VT positions in this paper. The algorithm is evaluated based on measurements in an indoor scenario with one fixed transmitter and a moving receiver. We show that indoor positioning is possible with only one transmitter when MPCs are used. 


\section{INTRODUCTION}

Positioning is next to communication the most important field of applications for wireless radio transmissions. Global navigation satellite system (GNSS) receivers can deliver very good position estimates under optimum conditions. Good accuracy can be achieved by ranging, i.e. the determination of the radio wave propagation distance from a transmitter at a known location to the receiver. Under pure line-of-sight (LoS) propagation conditions, the information of the wave's traveled distance can be extracted from the amplitude, phase or the delay of the wideband signal. To obtain the three dimensional position of the receiver, ranges to at least four different transmitters need to be measured, assuming that the transmitters are synchronized and their positions are known. However, in critical scenarios, the positioning accuracy of GNSS is reduced because of multipath effects, low received signal power and non-line-of-sight (NLoS) propagation. Though, signals of opportunity for example originated from mobile communication base-stations, dedicated ultra-wideband (UWB) transmitters or wireless local area network (WLAN) base-stations can be used for positioning. In this paper, we consider a positioning approach using wireless signals in challenging environments like indoor or deep urban. In such environments the transmitted signal is reflected and scattered by objects. Hence, the signal reaching the receive antenna consists of multiple paths, called multipath. Multipath reception degrades the accuracy of the positioning device as long as the receiver is based on standard methods. Optimal methods to mitigate multipath effects on the range estimate are based on the estimation of the channel impulse response where the first arrived path is treated as the LoS path. Therefore, these methods determine the multipath only to remove the influence on the range estimate of the LoS path.

With Channel-SLAM [1]-[4] we introduced a novel algorithm which uses multipath components (MPCs) for positioning instead of mitigating them. Measurements with a moving receive antenna showed, that some MPCs have a path life of several meters of the receiver movement [5]. These long visible paths can be used by Channel-SLAM for positioning. Channel-SLAM treats each MPC as a LoS signal from a virtual transmitter (VT) whose position is unknown to the receiver. These VTs are static during the receiver movement. ChannelSLAM estimates the receiver position and the positions of the VTs simultaneously, thus, contrarily to other approaches the approach does not require any prior information such as room-layout or a database for fingerprinting [6]-[8]. The only conditions to be fulfilled are the presence of a multipath environment, a moving receiver as well as prior knowledge of the initial receiver states, i.e. position and moving direction. To further improve the accuracy of Channel-SLAM, we fused in [4] additionally a gyroscope which provides heading information of the moving receiver.

This work builds on and extends the previous work on Channel-SLAM. Channel-SLAM basically uses a two level approach: The first level uses the Kalman enhanced super resolution tracking (KEST) algorithm to estimate and track the amplitude and the delay of each MPCs [9]. Afterwards, the second level estimates simultaneously the positions of the receiver and the VTs based on the estimated parameters of the MPCs. To track the receiver movement, we use a recursive Bayesian filter approach to estimate the probability density function of the receiver position and the VT positions. A method to estimate the position of the receiver at the same time as landmarks is called simultaneous localization and mapping (SLAM) [10]-[12]. Usually in robotics, SLAM covers the task of asserting whether the robot returned to a previously visited area, after moving for an arbitrary time which is called loop closure. This allows the robot to reuse previously gained information. Hence, we derived in [4] a probabilistic map representation based on previously visited locations. As soon as the receiver returns to an already mapped position, information of the previous movements at this position can be reused to obtain predictions for the further movement. In order to estimate and store the probability distribution of the receiver's motions as a function of location, we need to partition the space. Equivalently to [13]-[15], we propose a probabilistic map that represents the receiver positions by a two-dimensional hexagonal grid. In previous implementations of Channel-SLAM, we did not consider re-tracking previous MPCs or VTs. Hence, if the tracking of a MPC has been lost and might be regained, the corresponding VT is initialized without any prior information. Similar to SLAM approaches, we estimate, map and reuse VT positions in this paper. To verify the refined algorithm, we perform evaluations based on measurements. We used an indoor scenario with one fixed transmitter and a moving pedestrian, carrying the receive antenna and a gyroscope in his hands. The pedestrian was moving on the ground floor of a building with partly overlapping parts. Hence, we show that the algorithm is able to map the trajectory as well as reuse the estimated map and VTs.

The paper is structured as follows: Section II describes the signal model and its use by VTs, afterwards Section III derives the proposed algorithm. Thereafter, Section IV evaluates the algorithm based on measurements. The last section, Section V, concludes the paper.

Throughout the paper, we will use the following notation:

- $(\cdot)^{T},(\cdot)^{H}$ stands for matrix (or vector) transpose and conjugate transpose, respectively.

- All vectors are interpreted as column vectors.

- $\mathbf{x} \odot \mathbf{y}$ denotes the Hadamard-Schur product, i.e. the element-wise multiplication of vector $\mathbf{x}$ and $\mathbf{y}$.

- I denotes an identity matrix.

- Matrices are denoted by bold capital letters and vectors by bold small letters.

- $\|\mathbf{A}\|^{2}=\sum_{l} \sum_{m}\left|A_{l, m}\right|^{2}$ represents the square of the Frobenius norm of $\mathbf{A}$ with elements $A_{l, m}$.

- $a \sim \mathcal{N}\left(x ; \mu_{a}, \sigma_{a}^{2}\right)$ denotes a Gaussian distributed random variable $a$ with mean $\mu_{a}$ and variance $\sigma_{a}^{2}$.

- $1: k$ stands for all integer numbers starting from 1 to $k$, thus $1,2, \ldots, k$. 


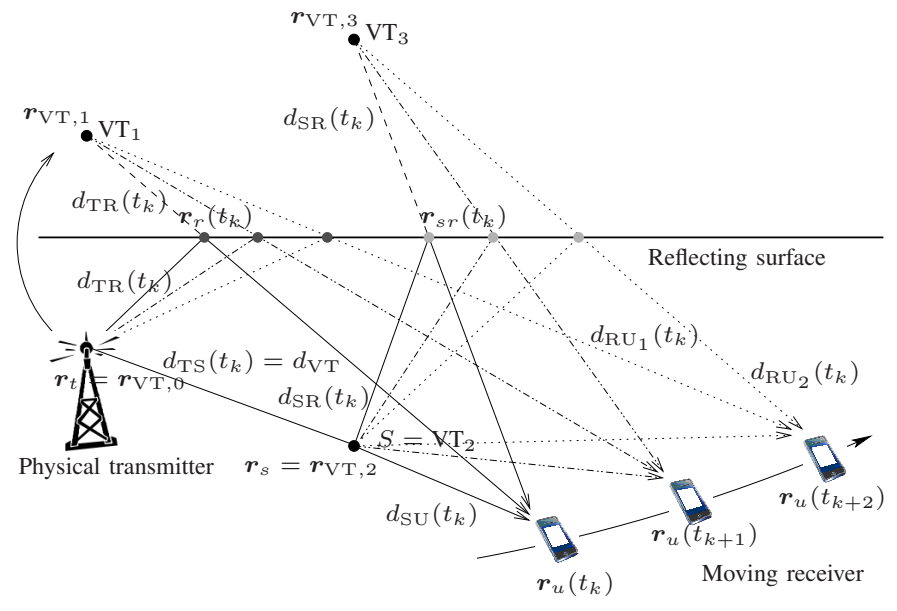

Fig. 1: The figure shows three propagation mechanism: First scenario: the transmitted signal is reflected on a smooth surface. $\mathrm{VT}_{1}$ is defined by mirroring the physical transmitter position at the surface. Second scenario: the transmitted signal is scattered at $S . \mathrm{VT}_{2}$ is defined at the position of $S$. Third scenario: The transmitted signal is scattered and afterwards reflected on a smooth surface. $\mathrm{VT}_{3}$ is defined by mirroring the scatterer $S$ at the surface. In the second and third scenario the additional propagation length $d_{\mathrm{VT}}$ equals to $d_{\mathrm{TS}}\left(t_{k}\right)$. Additional interactions between the physical transmitter and $S$ may occur indicated by the winded line.

- $\mathrm{p}(x)$ denotes the probability density function of $x$.

- $f^{-1}(\cdot)$ denotes the inverse of function $f(\cdot)$.

- $c$ is the speed of light.

\section{Signal Model}

Fig. 1 shows three propagation mechanism. In the first scenario, the transmitted signal is reflected on a smooth surface. The transmitter has a fixed position and the receiver is moving on the indicated path. When the receiver is moving also the reflection point at the coordinates $\boldsymbol{r}_{r}\left(t_{k}\right)$ is moving. If we mirror the physical transmitter position on the reflecting surface, we obtain the position $\boldsymbol{r}_{\mathrm{VT}, 1}$ of $\mathrm{VT}_{1}$ which is static during the receiver movement. The distance between $\mathrm{VT}_{1}$ and the receiver is equivalent to the propagation time of the reflected signal multiplied with the speed of light. Additionally, Fig. 1 exploits a scenario where the signal is scattered. The propagation effect of scattering occurs if an electromagnetic wave impinges an object and the energy is spread out in all directions. Geometrically, the effect of scattering can be described as a fixed point $S$ in the pathway of the MPC for all receiver positions. Hence, the propagation distance of the scattered path is $d_{\mathrm{TS}}\left(t_{k}\right)+d_{\mathrm{SU}}\left(t_{k}\right)=\left\|\boldsymbol{r}_{t}-\boldsymbol{r}_{s}\right\|+\left\|\boldsymbol{r}_{s}-\boldsymbol{r}_{u}\left(t_{k}\right)\right\|=$ $\left\|\boldsymbol{r}_{\mathrm{VT}, 2}-\boldsymbol{r}_{u}\left(t_{k}\right)\right\|+d_{\mathrm{VT}}$ where $d_{\mathrm{TS}}\left(t_{k}\right)=d_{\mathrm{VT}}>0$ is constant and $\boldsymbol{r}_{s}=\boldsymbol{r}_{\mathrm{VT}, 2}$ denotes the position of the scatterer. Thus, we define $S$ as $\mathrm{VT}_{2}$ for the MPC and treat $d_{\mathrm{VT}}>0$ as an additional propagation distance associated to the MPC. Furthermore, Fig. 1 shows a generalization of the considered multipath effects. The transmitted signal is scattered at $S$ and afterwards reflected. Between the transmitter and $S$ additional interactions are possible. When the receiver is moving, the reflection point on the reflecting surface is moving as well. Hence, the $\mathrm{VT}_{3}$ is defined by mirroring the scatterer $S$ at the surface as visualized.

To summarize, the propagation path of each MPC can be equivalently described as a direct path between a VT and the receiver plus an additional constant propagation length $d_{\mathrm{VT}}$. If only reflections occurred on the pathway between physical transmitter and receiver, this additional propagation length is zero $d_{\mathrm{VT}}=0$. If the MPC was interacting with a scatterer, the additional propagation length is greater than zero $d_{\mathrm{VT}}>0$. Equivalently, we can interpret $d_{\mathrm{VT}}$ as a constant clock offset between the VT and the physical transmitter. Hence, ChannelSLAM treats each MPC as a LoS signal from a VT with static position that is unknown to the receiver.

\section{Multipath Assisted Positioning Using Mapping}

The received signal is processed by the KEST algorithm, that estimates for each MPC $i$, the propagation distance $d_{i}\left(t_{k}\right)$. For each time instant $t_{k}$, the estimates of the KEST algorithm are condensed as the vector $\mathbf{z}\left(t_{k}\right)$ with

$$
\mathbf{z}\left(t_{k}\right)=\left[\tilde{d}_{0}\left(t_{k}\right), \ldots, \tilde{d}_{N\left(t_{k}\right)-1}\left(t_{k}\right)\right]^{T},
$$

where $N\left(t_{\tilde{\sigma}}\right)$ denotes the number of MPCs and the KEST estimates $\tilde{d}_{i}\left(t_{k}\right)$ of $d_{i}\left(t_{k}\right)$. As introduced in [4], in a Bayesian formulation, the posterior probability density function (PDF) is defined by

$$
\mathrm{p}\left(\mathbf{x}\left(t_{0: k}\right), \mathbf{M}\left(t_{0: k}\right) \mid \mathbf{z}\left(t_{0: k}\right)\right)
$$

where $\mathbf{M}\left(t_{0: k}\right)$ defines the probabilistic map of the previous receiver trajectory and $\mathbf{x}\left(t_{0: k}\right)$ defines the state vector. Here, the state vector $\mathbf{x}\left(t_{k}\right)$ at time instant $t_{k}$ for $N\left(t_{k}\right)$ MPCs is defined by

$$
\mathbf{x}\left(t_{k}\right)=\left[\mathbf{x}_{u}\left(t_{k}\right)^{T}, \mathbf{x}_{\mathrm{VT}, 0}\left(t_{k}\right)^{T}, \ldots, \mathbf{x}_{\mathrm{VT}, N\left(t_{k}\right)-1}\left(t_{k}\right)\right]^{T}
$$

with the receiver state

$$
\mathbf{x}_{u}\left(t_{k}\right)=\left[\boldsymbol{r}_{u}\left(t_{k}\right)^{T}, \boldsymbol{v}_{u}\left(t_{k}\right)^{T}, b_{u}\left(t_{k}\right), \rho_{u}\left(t_{k}\right)\right]^{T}
$$

where $\boldsymbol{r}_{u}\left(t_{k}\right)$ is the receiver position, $\boldsymbol{v}_{u}\left(t_{k}\right)$ the receiver velocity, $b_{u}\left(t_{k}\right)$ and $\rho_{u}\left(t_{k}\right)$ the receiver's clock bias and drift, respectively. The parameters representing the VT of the $i$-th MPC are defined as

$$
\mathbf{x}_{\mathrm{VT}, i}\left(t_{k}\right)=\left[\boldsymbol{r}_{\mathrm{VT}, i}\left(t_{k}\right)^{T}, d_{\mathrm{VT}, i}\left(t_{k}\right)\right]^{T},
$$

where $\boldsymbol{r}_{\mathrm{VT}, i}\left(t_{k}\right)$ are the coordinates of the $\mathrm{VT}$ and $d_{\mathrm{VT}, i}\left(t_{k}\right)$ the additional propagation distance. We can factorize (2) into

$$
\begin{aligned}
& \mathrm{p}\left(\mathbf{x}\left(t_{0: k}\right), \mathbf{M}\left(t_{0: k}\right) \mid \mathbf{z}\left(t_{0: k}\right)\right) \\
& =\mathbf{p}\left(\mathbf{x}\left(t_{0: k}\right) \mid \mathbf{z}\left(t_{0: k}\right)\right) \cdot \mathbf{p}\left(\mathbf{M}\left(t_{0: k}\right) \mid \mathbf{z}\left(t_{0: k}\right), \mathbf{x}\left(t_{0: k}\right)\right) \\
& =\underbrace{\mathbf{p}\left(\mathbf{x}\left(t_{0: k}\right) \mid \mathbf{z}\left(t_{0: k}\right)\right)}_{\text {Channel-SLAM }} \cdot \underbrace{\mathbf{p}\left(\mathbf{M}\left(t_{0: k}\right) \mid \mathbf{x}_{u}\left(t_{0: k}\right)\right)}_{\text {mapping problem conditioned on the receiver state }}
\end{aligned}
$$

where we assume that the map $\mathbf{M}\left(t_{k}\right)$ only depends on the receiver state $\mathbf{x}_{u}\left(t_{k}\right)$ as part of $\mathbf{x}\left(t_{k}\right)$. In (6), the first term 


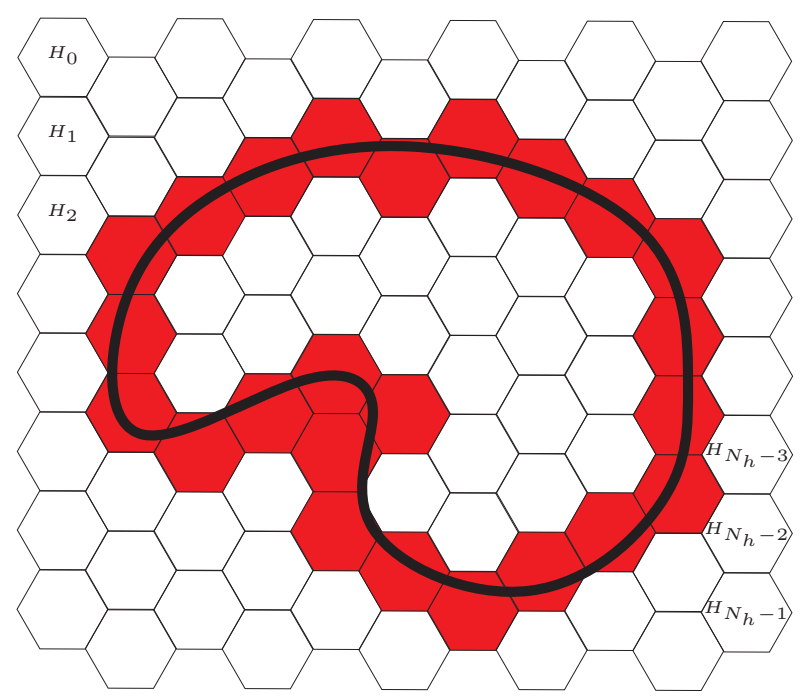

Fig. 2: Representing a walked path by hexagons: the black line indicates the walked path, the red hexagons shows the mapped path.

$\mathrm{p}\left(\mathbf{x}\left(t_{0: k}\right) \mid \mathbf{z}\left(t_{0: k}\right)\right)$ defines the posterior of Channel-SLAM described in [1] and the second term $\mathrm{p}\left(\mathbf{M}\left(t_{0: k}\right) \mid \mathbf{x}_{u}\left(t_{0: k}\right)\right)$ defines the probabilistic map described in [4].

As mentioned in [4], to estimate and store the probability distribution of receivers motions as a function of location, we need to partition the space. We discretize the space into a grid of $N_{h}$ adjacent hexagons $H_{i} \in\left\{H_{0}, H_{1}, \ldots, H_{i}, \ldots H_{N_{h}-1}\right\}$, where $i$ uniquely references a position of a hexagon. Fig. 2 shows an example where the walked path is indicated by the black line, the corresponding discretized hexagon map is indicated in red. We define by $\mathbf{M}\left(t_{k}\right)$, the time invariant map of the previous receiver positions discretized by hexagons.

In previous implementations of Channel-SLAM, we did not consider re-tracking previous MPCs or VTs. Hence, if the tracking of a MPC has been lost and might be regained, the corresponding VT is initialized without any prior information. Similar to SLAM approaches, we estimate, map and reuse VT positions in this paper. Thus, additional information on regions from where each VT is visible has to be included in the hexagon map. Before the receiver returns to a previously mapped position (first loop), the algorithm initializes all VTs entirely new, without a-priori information. Hence, each VT is estimated in a discrete representation of the posterior probability density function of the VT states, by particle clouds, see [1]. This part of the algorithm has high computational complexity because the receiver has no information about the states and hence, the location of the VTs. During the receiver movement, the posterior probability density function of the VT state converges which reduces the computational complexity. In order to reuse the estimated VT locations, we save for each mapped hexagon $H_{i}$ the estimated VT locations. When the receiver returns to a previously mapped hexagon $H_{i}$ (loopclosure) the algorithm evaluates the similarities among all VTs. Therefore, the algorithm can reuse the estimated VTs for positioning when the receiver returns to an already mapped position. This enables on the one hand a lower complexity of the algorithm as well as a better position estimation of the receiver position.

\section{MeAsurements}

This section evaluates the derived algorithm based on indoor channel measurements with a single static physical transmitter and a moving pedestrian as shown in Fig. 3 and Fig. 4. The pedestrian moves on the blue track for $543 \mathrm{~s}$, moving from the parking lot inside the building, down two corridors, inside the meeting room and back out to the parking lot. For the following analyzes, we divide the track into different sections, see Table I. The measurements were performed using the MEDAV RUSK-DLR broadband channel sounder in single-input single-output (SISO) mode with the measurement parameters as summarized in Table II. As shown in Fig. 5, the moving pedestrian was equipped with the receive antenna and a Xsense inertial measurement unit (IMU). We captured simultaneously the received signal as well as the turn rates of the gyroscope of the IMU. To measure the coordinates we use a tachymeter TPS1200 from Leica Geosystems AG. We mounted a prism as shown in Fig. 5 next to the receive antenna on a stick above the moving person. The tachymeter was located in the lobby where the prism of the moving pedestrian is in LoS conditions to the tachymeter in the sections I, II, IV, VI, VII and VIII. For the corridor parts, sections III and $\mathrm{V}$, the reference position was obtained using previously measured ground truth points. The tachymeter transmits the measured coordinates to the channel sounder which records the channel impulse responses (CIRs) and the coordinates simultaneously. During the walk the pedestrian is moving with a constant speed, except between the time instants $230 \mathrm{~s}$ and $285 \mathrm{~s}$, and between $395 \mathrm{~s}$ and $415 \mathrm{~s}$, the pedestrian was standing. Fig. 6 shows the recorded unprocessed CIRs versus the receiver traveled distance in seconds. The figure shows the delay multiplied by the speed of light in meter. It is apparent that this is a high multipath scenario with path delays up to $100 \mathrm{~m}$. Many MPCs are closely spaced regarding their delay, nevertheless for some parts of the track it is possible to determine the evolution of different MPCs by inspection. The plot also shows that we are able to receive a signal from the physical transmitter all the time. There are periods with high power, when the user is in the lobby close to the physical transmitter, and periods with low power when the LoS is blocked or attenuated, e.g. outdoors or inside the corridors.

In order to exploit the multipath propagation for positioning, we have to estimate and track the MPCs over time. Hence, the accuracy of Channel-SLAM relies directly on the accuracy of the CIR estimations of KEST. Fig. 7 shows the estimation results of KEST for the CIR versus the receiver traveled time in seconds. Many paths can be tracked for several seconds of receiver movement. Channel-SLAM considers an underdetermined system, therefore, long visible paths are preferable. Thus for the evaluations, we extract from Fig. 7 only the long visible paths as visualized in Fig. 8. Anyhow, Channel-SLAM 


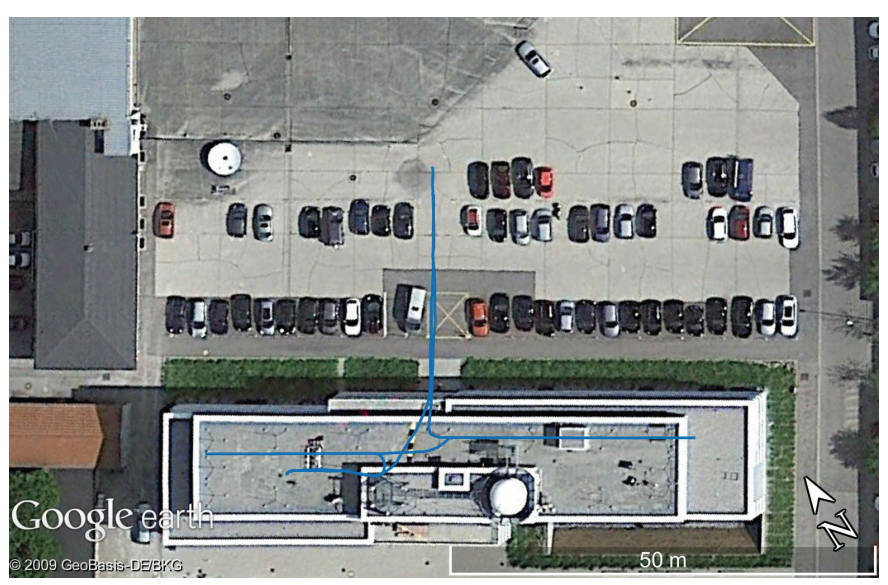

Fig. 3: Satellite Image: The pedestrian is moving from the parking lot inside the building, down two corridors, inside the meeting room and back out to the parking lot.

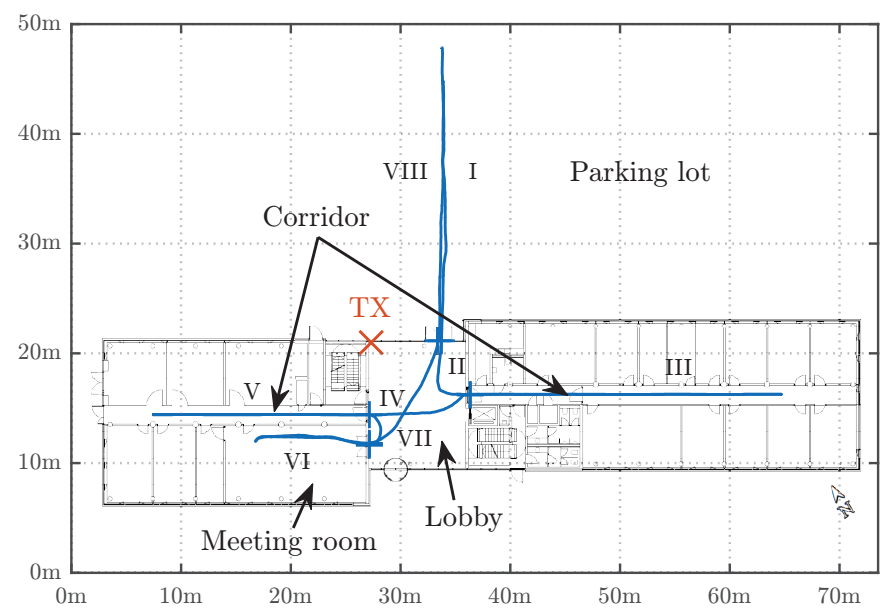

Fig. 4: Measurement scenario with a fixed transmitter and a moving receiver (pedestrian). The pedestrian moves on the blue track for $543 \mathrm{~s}$, moving from the parking lot inside the building, down two corridors, inside the meeting room and back out to the parking lot. The track is divided into different sections, see Table I.

TABLE I: Description of the different sections of the track

\begin{tabular}{lll}
\hline Section & Beginning at & Description \\
\hline I & $t=0 \mathrm{~s}$ & Outdoor \\
\hline II & $t=62 \mathrm{~s}$ & Lobby \\
\hline III & $t=93 \mathrm{~s}$ & Right corridor \\
\hline IV & $t=233 \mathrm{~s}$ & Lobby transition \\
\hline V & $t=315 \mathrm{~s}$ & Left corridor \\
\hline VI & $t=404 \mathrm{~s}$ & $\begin{array}{l}\text { Short lobby passage } \\
\end{array}$ \\
\hline VII & $t=490 \mathrm{~s}$ & L meeting room \\
\hline VIII & $t=518 \mathrm{~s}$ & Outdoor \\
\hline
\end{tabular}

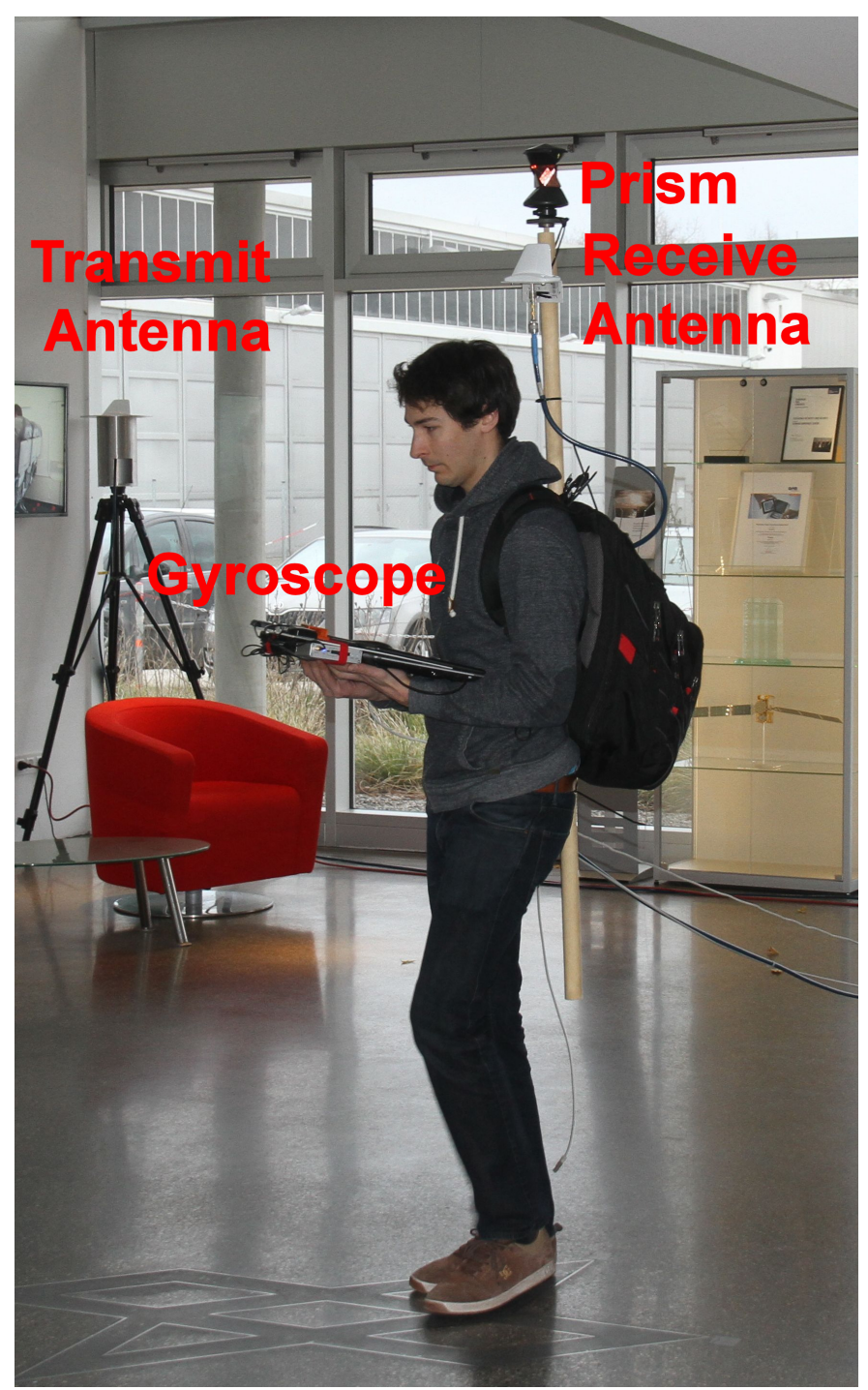

Fig. 5: Moving pedestrian: The receive antenna mounted on a stick next to a prism for measuring the ground truth of the moving pedestrian. The gyroscope is carried in the hands of the pedestrian.

could use all detected MPCs, however, this would increase the computational complexity.

Fig. 8 shows only the paths of KEST which are tracked for a long receiver movement. The black dashed line in Fig. 8 shows the geometrical line-of-sight (GLoS) path. For the first section of the track (I), we have LoS condition, where the signal of the LoS path is attenuated by the coated window. We are walking towards the physical transmitter, so the delay of the LoS path decreases. The other paths behave similarly, indicating their corresponding VT lies in the moving direction. When we enter the lobby (II), the received power increases and drops again by the time we enter the first corridor (III). During the movement in the corridor, one MPC with a slightly larger delay than the calculated LoS is present, which is probably caused by a diffraction at the entrance door of the corridor. 
TABLE II: Channel sounder settings

\begin{tabular}{ll}
\hline Parameter & Value \\
\hline RF center frequency & $1.51 \mathrm{GHz}$ \\
Bandwidth $B$ & $100 \mathrm{MHz}$ \\
Number of sub-carriers $N$ & 1281 \\
Sub-carrier spacing $\Delta f$ & $78.125 \mathrm{kHz}$ \\
Transmit power & $10 \mathrm{~mW}$ \\
Signal period $T_{p}$ & $12.8 \mu \mathrm{s}$ \\
Measurement rate $T_{g}$ & $1.024 \mathrm{~ms}$ \\
Transmitter antenna & Omni-directional (V-polarized) \\
Receiver antenna & Omni-directional (V-polarized) \\
\hline
\end{tabular}

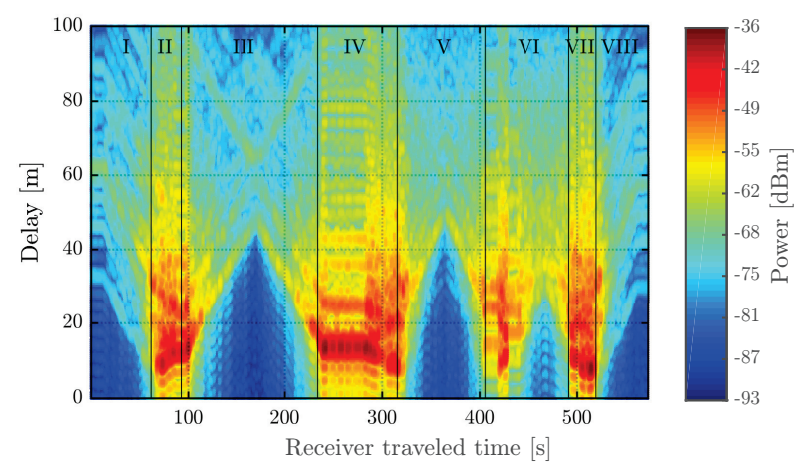

Fig. 6: Recorded unprocessed CIRs versus the receiver traveled distance in seconds. The track is divided into different sections, see Table I.

Interestingly for this part of the track we also have a signal originated from the opposite direction, which is apparently being reflected at the end of the corridor. As expected, the power rises again when we leave the corridor, stand still for some time and then cross the lobby (IV). It stands out that the LoS component is tracked rather inaccurately for this part. This might be caused by limited bandwidth and closely spaced MPCs. Hence KEST is not able to resolve all paths properly and the estimated LoS path is not identical to the geometrical LoS path. Inside the second corridor (V), we obviously have a NLoS condition. After shortly passing the lobby we enter the meeting room (VI). Apparently this is a challenging situation for KEST. Although several MPCs are present, they can only be tracked for a short period of time. Finally, we walk out of the meeting room and leave the building through the lobby (VI). Outdoors (VIII) we roughly take the same way as in the beginning, but stop $3 \mathrm{~m}$ after the starting point. Comparing section I and VIII, we can see that the evolution of the MPCs is similar, however not all MPCs are tracked in both sections.

Fig. 9, Fig. 10, Fig. 11 and Fig. 12 illustrate the performance of the derived algorithm. For simplicity we show in these figures only the estimations of the pedestrian position and the mapped paths. We show by the green circles the particle filter $(\mathrm{PF})$ estimations of the receiver position and by the red circle the minimum mean square estimate, see [1], of

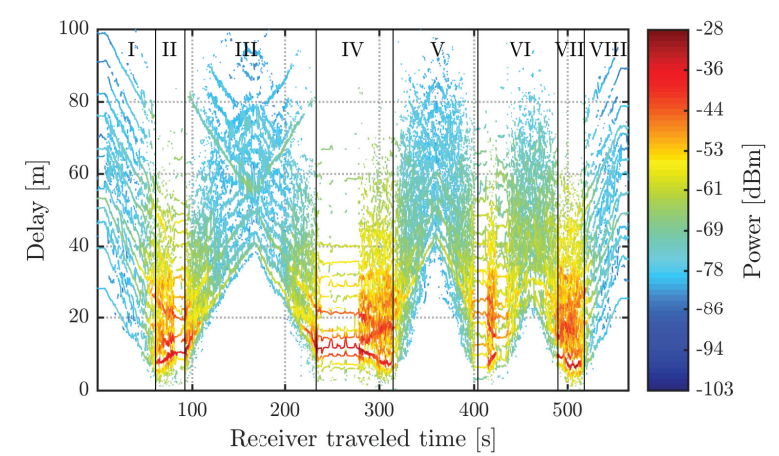

Fig. 7: Estimation results of KEST for the CIR versus the receiver traveled distance in meters. The track is divided into different sections, see Table I.

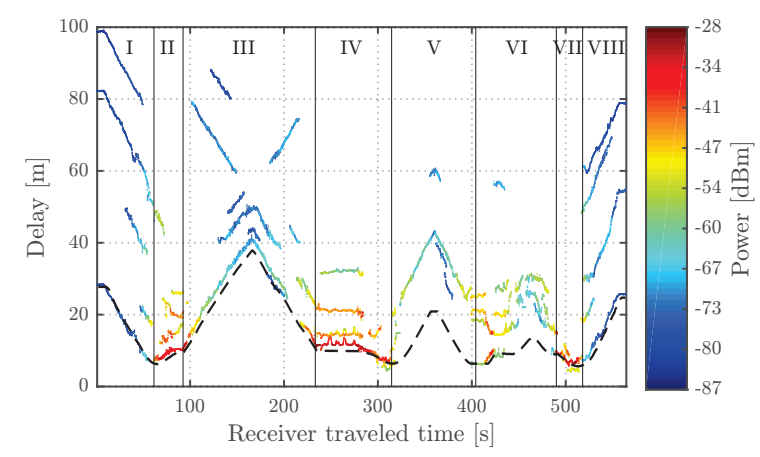

Fig. 8: Estimation results of KEST for the CIR versus the receiver traveled distance in meters. Only long tracked paths are visualized. The black dashed line indicates the GLoS path. The track is divided into different sections, see Table I.

the pedestrian position. The estimations of the VT locations are not shown in this paper, example of the estimations can be found e.g. in [4]. Channel-SLAM does not need any prior information except of a coarse receiver starting position. Hence, the receiver position is initialized in a square with $1 \mathrm{~m} \times 1 \mathrm{~m}$ around the correct starting position, see Fig. 9. For the evaluations of Channel-SLAM, we do not assume any prior knowledge on the transmitters, i.e. we estimated the positions of the physical transmitter and virtual transmitter simultaneously. Similarly to Fig. 9, Fig. 10 shows the estimation results at $t_{k}=144 \mathrm{~s}$ at the end of the corridor of section III. The figure shows the mapped hexagons, where the grey hexagons indicate the estimated trajectory with the highest (particle) weight. After turning at the end of the corridor, the probabilistic map enhances the movement prediction until we reached the lobby again, see Fig. 11. We see that the uncertainty of the position estimation of the receiver is reduced. Fig. 12 shows the estimation results at the end of the track. We can observe, that we are able to map the receiver path accurately. We see that during the receiver movement the uncertainty on the estimated pedestrian position increases. However, due to the mapping and reusing of the map, we are able estimate the pedestrian trajectory with an error smaller than $3 \mathrm{~m}$ for the 
00:00:00

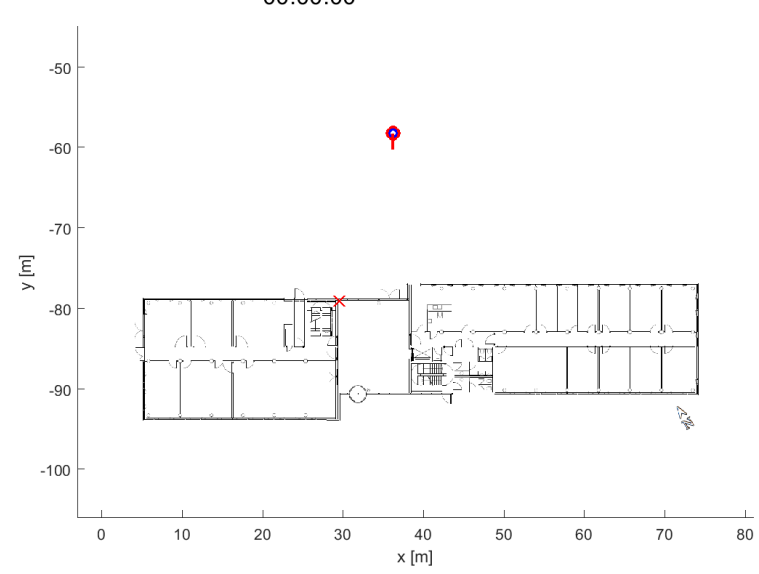

Fig. 9: Initialization of the algorithm at $t_{k}=0 \mathrm{~s}$.

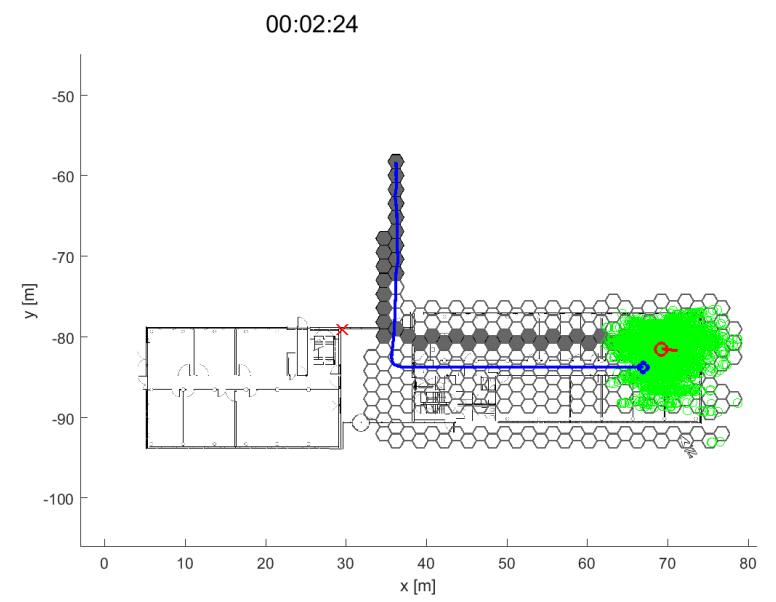

Fig. 10: Estimated receiver position and map after $t_{k}=144 \mathrm{~s}$ at the end of the corridor.

whole scenario.

\section{CONCLUSIONS}

In this paper, we presented and extended the work on multipath assisted positioning, called Channel-SLAM. The new positioning method uses a SLAM approach to map the receiver position. We propose a probabilistic map that represents the receiver motion in a two-dimensional hexagonal grid. Hence, as soon as the receiver returns to an already mapped position, information of this position can be reused to obtain better position estimations of the receiver as well as the VT positions. Additionally, the obtained maps can be shared between different receivers and VTs can be used similarly to real transmitters with the advantage that VTs are always in LoS to the receiver. We evaluated the algorithm based on indoor measurements with a moving pedestrian and one fixed transmitter.

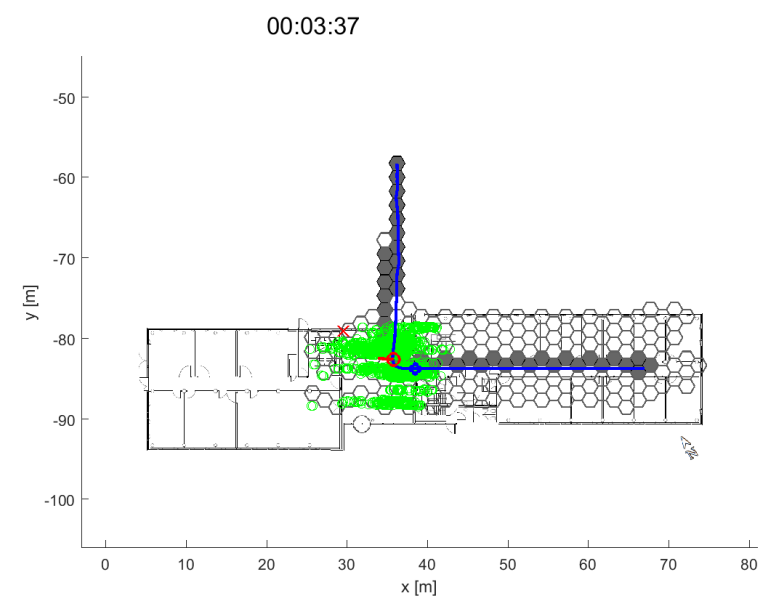

Fig. 11: Estimated receiver position and map after $t_{k}=217 \mathrm{~s}$ entering the lobby.

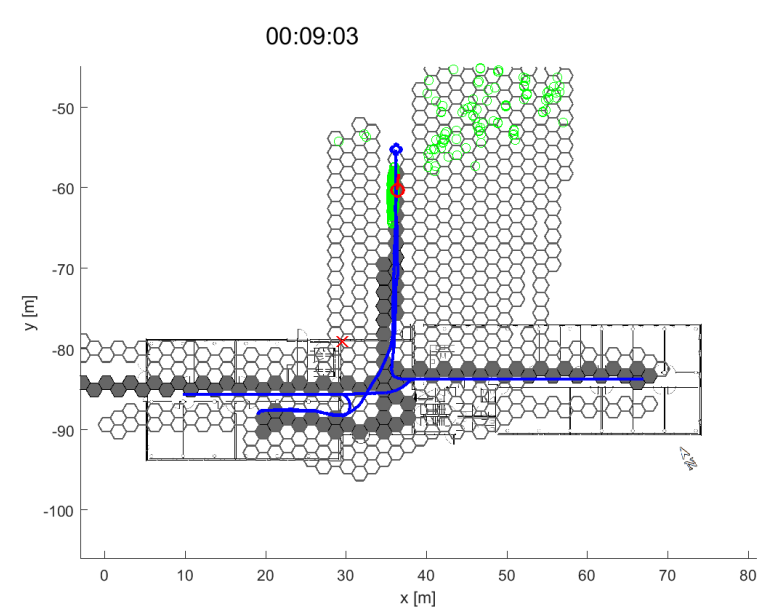

Fig. 12: Estimated receiver position and map after $t_{k}=543 \mathrm{~s}$ at the end of the track.

\section{ACKNOWLEDGEMENT}

This work has been performed in the framework of the DLR project Dependable Navigation and the European Unions Horizon 2020 research and innovation programme under grant agreement No. 636537 HIGHTS (High precision positioning for Cooperative-ITS applications).

\section{REFERENCES}

[1] C. Gentner, T. Jost, W. Wang, S. Zhang, A. Dammann, and U.-C. Fiebig, "Multipath Assisted Positioning with Simultaneous Localization and Mapping," IEEE Trans. Wireless Commun., vol. 15, no. 9, pp. 61046117, Sep. 2016.

[2] C. Gentner and T. Jost, "Indoor Positioning using Time Difference of Arrival between Multipath Components," Montbeliard, France, Oct. 2013.

[3] C. Gentner, R. Pöhlmann, M. Ulmschneider, T. Jost, and A. Dammann, "Multipath Assisted Positioning for Pedestrians," Tampa, FL, USA, Sep. 2015. 
[4] C. Gentner, B. Ma, M. Ulmschneider, T. Jost, and A. Dammann, "Simultaneous Localization and Mapping in Multipath Environments," in Accepted at IEEE/ION PLANS, Savanah, GO, USA, Apr. 2016.

[5] T. Jost, W. Wang, U.-C. Fiebig, and F. Pérez-Fontán, "A Satellite-toIndoor Channel Model: Multipath Components," Prague, Czech Rep., Mar. 2012.

[6] M. Triki, D. Slock, V. Rigal, and P. Francois, "Mobile Terminal Positioning via Power Delay Profile Fingerprinting: Reproducible Validation Simulations," Sep. 2006, pp. 1-5.

[7] E. Kupershtein, M. Wax, and I. Cohen, "Single-Site Emitter Localization via Multipath Fingerprinting," IEEE Trans. Signal Process., vol. 61, no. 1, pp. 10-21, Jan. 2013

[8] Y. Shen and M. Win, "On the Use of Multipath Geometry for Wideband Cooperative Localization," Dec. 2009, pp. 1-6.

[9] T. Jost, W. Wang, U.-C. Fiebig, and F. Pérez-Fontán, "Detection and Tracking of Mobile Propagation Channel Paths," IEEE Trans. Antennas Propag., vol. 60, no. 10, pp. 4875-4883, Oct. 2012.

[10] R. Smith and P. Cheeseman, "On the Representation and Estimation of Spatial Uncertainty," vol. 5, no. 4, pp. 56-68, 1986.

[11] J. Leonard and H. Durrant-whyte, "Simultaneous Map Building and Localization For an Autonomous Mobile Robot," in Proc. IEEE/RSJ Int. Workshop on Intelligent Robots and Systems, Osaka, Japan, Nov. 1991.

[12] H. Durrant-Whyte and T. Bailey, "Simultaneous Localization and Mapping: Part I," IEEE Robot. Autom. Mag., vol. 13, no. 2, pp. 99-110, Jun. 2006.

[13] P. Robertson, M. Angermann, and B. Krach, "Simultaneous Localization and Mapping for Pedestrians using only FootMounted Inertial Sensors," in In Proc. UbiComp 2009, ACM, 2009, pp. 93-96.

[14] M. Angermann and P. Robertson, "FootSLAM: Pedestrian Simultaneous Localization and Mapping Without Exteroceptive Sensors - Hitchhiking on Human Perception and Cognition," Proceedings of the IEEE, vol. 100, no. Special Centennial Issue, pp. 1840-1848, May 2012.

[15] M. Puyol, D. Bobkov, P. Robertson, and T. Jost, "Pedestrian Simultaneous Localization and Mapping in Multistory Buildings Using Inertial Sensors," Intelligent Transportation Systems, IEEE Transactions on, vol. 15, no. 4, pp. 1714-1727, Aug 2014. 